\title{
Tribochemical Purification of Gases. I. The Process Model
}

\author{
Konstantin Chuntonov ${ }^{1}$ (D), Alexey O. Ivanov², Viktor L. Kozhevnikov ${ }^{3}$ \\ ${ }^{1}$ Mechemlab Ltd., Nesher, Israel \\ ${ }^{2}$ Institute of Mathematics and Computer Sciences, Ural Federal University, Yekaterinburg, Russia \\ ${ }^{3}$ Institute of Solid State Chemistry, Russian Academy of Sciences, Yekaterinburg, Russia \\ Email: konstantin@chuntonov.com
}

How to cite this paper: Chuntonov, K., Ivanov, A.O. and Kozhevnikov, V.L. (2020) Tribochemical Purification of Gases. I. The Process Model. Journal of Materials Science and Chemical Engineering, 8, 37-54. https://doi.org/10.4236/msce.2020.82005

Received: December 17, 2019

Accepted: January 19, 2020

Published: January 22, 2020

Copyright (c) 2020 by author(s) and Scientific Research Publishing Inc. This work is licensed under the Creative Commons Attribution International License (CC BY 4.0). http://creativecommons.org/licenses/by/4.0/

\begin{abstract}
A mathematical model of the sorption process in a tribochemical reactor with a stirrer, where monolithic granules of alkali-earth metals mutually rub in the media of the flow gas, has been built. The products of reaction of the metal with the gas impurity are continuously removed mechanically from the surface of the granules, creating new metallic regions. As a result the total area of the sorbing surface appears to be by orders of magnitude larger than that of the traditional getter materials of the same mass in the existing sorption technologies. It follows from the model that the gas purification process obeys the linear law at a constant rotation speed of the stirrer. This dependence results in a great simplification of the process control. It also makes possible replacement of a periodic mode of operation by a continuous one where the sorption capacity of the consumed chemisorbent attains theoretical limit.
\end{abstract}

\section{Keywords}

Tribochemical Reactor, Sorption, Mathematical Model, Gases, Purification

\section{Introduction}

Powders, films or gas-permeable porous bodies of alkali-earth metal alloys are effective chemisorbents [1]-[6]. They react with active gases at room temperature and capture them in a form of nonvolatile chemical compounds. Not only the surface of the metal is available for these reactions with gases but its volume as well thus providing a great advantage of the alkali-earth metal alloys over adsorbents with respect to the specific sorption capacity [7] [8] [9]. To underline this peculiarity of the sorption behavior of the reactive alloys it was suggested to call them "reactants" and to single them out into a separate class of getter mate- 
rials [10].

While the sorption capacity defines the amount of the consumed in the production process chemisorbent, its other characteristics, the sorption rate, defines time consumption. Taken together, these two values point at the potential capabilities of the purification material, although a lot depends also on the production conditions in which the given material is used. Therefore, the analysis of all factors influencing the sorption process is needed in order to find optimal regimes for gas purification.

The aim of the present work is to describe the gas purification technology based on the use of cast granules of getter reactants in a flow reactor with a stirrer [11]. We outline the model of an ideal sorption column with a getter reactant as a consumable sorption material. Experimental results on gas sorption by reactants in the process of their mechanical activation are presented in the publication to follow.

\section{Reactive Sorber}

The reactive sorber is a triboreactor for removal of impurities from a gas flow by cast granules of the getter reactant at stirring [11]. This sorption process differs from the usual arrangement of tribochemical reactions [12] [13] [14] [15]. Due to high chemical activity and small mechanical strength of alkali-earth metal reactants there is no need for subjecting reactant granules to impingement attack. In order to maintain a reaction between the metal and the gas at the required level, a slight mixing of the granules with a stirrer is enough.

In this relation we have to notice that while in conventional mechanochemistry one of the central questions of study is the question about quantitative relation between the reaction rate and the consumed power, in consideration of sorption gas purification technologies it is more convenient to shift the attention to the dependence of the form $c=f(t)$, where $c$ is the concentration of gas impurity in the outlet product and $t$ is the time of the tribochemical treatment.

\subsection{The Problem Statement}

The design of the sorber is shown in Figure 1.

The initial gas is fed from above into the triboreactor 1. Then it passes through the column of stirred with the stirrer 2 granules of reactant 4 and exits from the column in the purified form below the dividing mesh 5 . The formed on the surface of the granules products of the reaction between the gas impurity and the metal fall at the mutual rubbing of the granules and are taken away with the gas in the form of submicron and/or micron particles through the openings in the mesh 5 into the waste collector 7 . The problem is to define how the impurity concentration $c$ in the outlet gas flow changes with time.

Two factors are to the greater degree than the others responsible for the run and the specific character of the sorption process in the given method. The first one is the shape of the blades of the stirrer 2 (Figure 1) that creates an upward-directed constituent in the trajectory of the moving granules. This constituent 


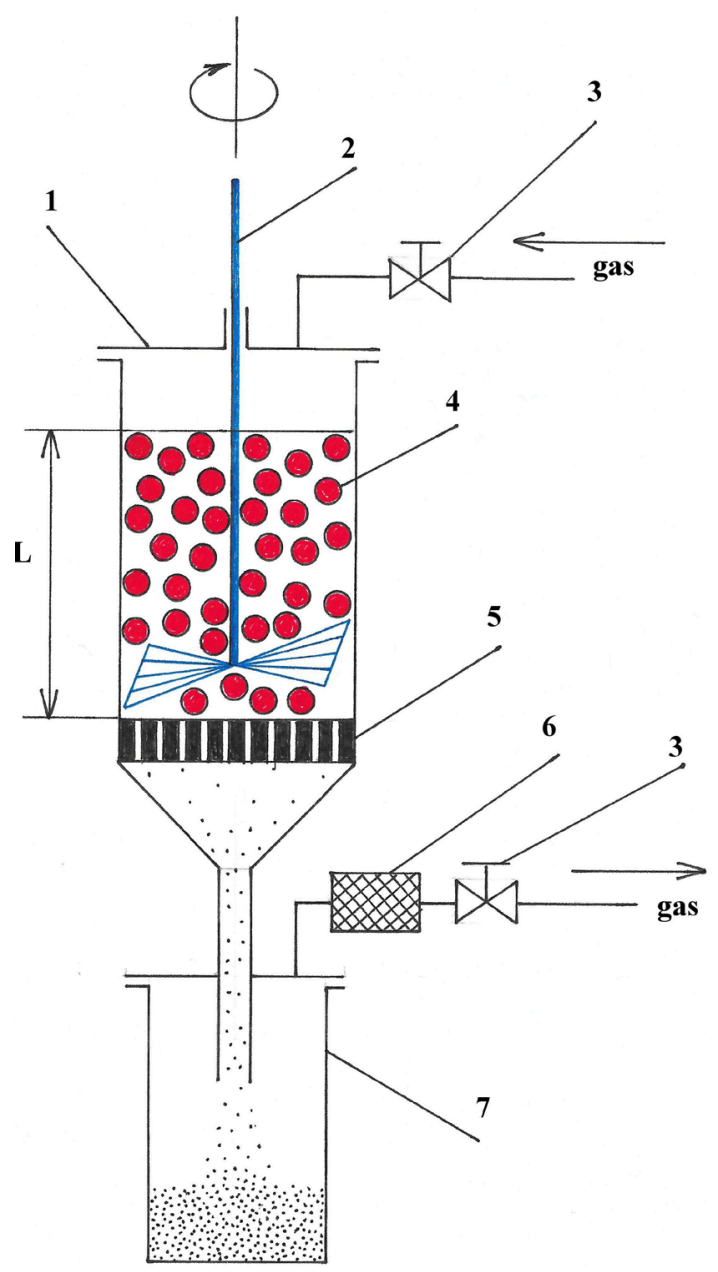

Figure 1. The sketch of reactive sorber. 1-triboreactor, 2-stirrer, 3-gas valve, 4-reactant, 5-dividing mesh, 6-filter, 7-waste collector.

leads to the averaging of the parameters of the reactant along the height of the column. The second factor is the number of rotations $\omega$ of the stirrer per time unit. Increasing $\omega$ from zero to a certain value it is possible to make the sorber with the getter reactant go through all the possible gas purification regimes from the case with the motionless mass of the granules, the surface of which is covered with the layer of growing products [16] to the extreme variant of the tribochemical treatment of granules, when their entire surface is free from the products of reaction.

Here we will be interested in the intermediate regime of gas purification, when part of the surface of the granules is covered with the products of reaction and part is free from them. The given choice is grounded in the fact that the rate of capturing gases by the metal surface is by orders of magnitude higher than by the surface with the products of reaction, where the process kinetics is limited by diffusion through the layer of these products. Even when the share of the metallic regions $\gamma$ is small the sorption contribution of the rest of the surface can be neglected. And this fact has a decisive importance: changing the rotation speed $\omega$ 
of the stirrer it is possible to control the number $\gamma$ and thus the entire sorption process.

\subsection{Mathematical Model}

Let the sorber be filled with cast granules of reactant Me forming a cylindrical column of purification material with radius $R$ and height $L$. The gas, which is fed from above, contains impurity $\mathrm{Y}$, the concentration of which is $c$. The gas flow rate at the entrance into the column of granules Me is equal to $v$. Then the gas flows along the voids between the granules of radius $r$ and reacts with $\mathrm{Me}$ forming the solid product MeY.

In the column of the reactant Me let us single out an elementary layer bounded by the cross-sections $x_{1}$ and $x_{2}=x_{1}+\mathrm{d} x$, that is perpendicular to the vertical axis $x$ of the column Me. The number $N_{\mathrm{Y}}$ of molecules $\mathrm{Y}$ in the given layer by the moment of time $t$ is

$$
N_{\mathrm{Y}}(t, x)=c(t, x) \frac{\pi \varepsilon P R^{2} \mathrm{~d} x}{k_{\mathrm{B}} T},
$$

where $c(t, x)$ is the fraction of the impurity molecules in the gas phase, $k_{\mathrm{B}}$ is the Bolzmann constant, $P$ is the gas pressure, $\mathcal{E}-$ is the porosity of the column of granules $\mathrm{Me}$, and $T$ is the temperature. Then the equation for numerical balance of molecules $\mathrm{Y}$ will be written in the form

$$
\frac{\mathrm{d} N_{\mathrm{Y}}}{\mathrm{d} t}=J_{\mathrm{Y}}\left(t, x_{1}\right)-J_{\mathrm{Y}}\left(t, x_{2}\right)-Q_{\mathrm{Y}}(t, x) \mathrm{d} x,
$$

where $J_{\mathrm{Y}}$ is the flow of molecules $\mathrm{Y}$, entering the layer $\mathrm{d} x$ at the boundary $x_{1}$ and exiting at the boundary $x_{2}$, and $Q_{\mathrm{Y}}$ is the rate of sorption of the impurity in the middle cross-section of the elementary layer. Basing on the results of the analysis of gas flows in the tubes with granular reactants [16] it is possible to write down Equation (2) in a more compact form

$$
\varepsilon \frac{\partial c}{\partial t}+v \frac{\partial c}{\partial x}=-Q_{\mathrm{Y}}(t, x) \frac{k_{\mathrm{B}} T}{P \pi R^{2}} .
$$

In Equation (3) the uncertainty in respect to the value $Q_{\mathrm{Y}}$, which is responsible for the "chemical sink" of the impurity Y is reserved. In order to disclose $Q_{\mathrm{Y}}$ it is necessary to formulate the sorption law for the reactant in the conditions of stirring the granules Me. According to the said in the previous chapter let us assume that capturing of the molecules of $\mathrm{Y}$ occurs practically only in the regions of the surface, which are free from the products of reaction. This assumption will be knowingly fulfilled if we consider the number of molecules $Y$, captured by the elementary layer during the time $\mathrm{d} t$, to be equal to the product $\gamma k_{0} S_{\mathrm{Me}} c(t, x) P \mathrm{~d} t / k_{\mathrm{B}} T$, where $k_{0}$ is the coefficient of sorption of the impurity by the pure surface $\mathrm{Me}$, and the surface area $S_{\mathrm{Me}}$ of the granules of the elementary layer is $3 \pi(1-\varepsilon) R^{2} \mathrm{~d} x / r(t)$. In this case we get for the "sink" summand in Equation (3)

$$
Q_{\mathrm{Y}}(t, x) \frac{k_{\mathrm{B}} T}{P \pi R^{2}}=(1-\varepsilon) \gamma k_{0} \frac{3 c(t, x)}{r(t)},
$$


where the dependence of $r$ on $t$ is expressed in the following way (see Appendix, formula A4)

$$
\frac{\mathrm{d} r}{\mathrm{~d} t}=-b \frac{c(t, x)}{c_{0}}, b=\gamma k_{0} c_{0} \frac{M_{\mathrm{Me}}}{\rho_{\mathrm{Me}} N_{\mathrm{A}}} \frac{P}{k_{\mathrm{B}} T} .
$$

Here $c_{0}$ is the concentration of the impurity in the entering into the sorber gas, $\rho_{\mathrm{Me}}$ is the density of the reactant Me, $M_{\mathrm{Me}}$ is the molar mass of the reactant and $N_{\mathrm{A}}$ is Avogadro number.

Let us now pass over from the elementary layer $\mathrm{d} x$ to the entire mass of granules Me. The concentration $c(t, x)$ of the impurity $\mathrm{Y}$ in the gas flow changes both with time and along the height of the column Me while the size of the granules due to their stirring in the entire volume of the purification material depends only on time. This gives us the right to perform averaging of the granules radius along the entire column Me using the expression

$$
\frac{\mathrm{d} r}{\mathrm{~d} t}=-\frac{b}{L(t)} \int_{0}^{L(t)} \frac{c(t, x)}{c_{0}} \mathrm{~d} x,
$$

where $L(t)$ is the current height of the column Me.

As the result, the mathematical model of the sorption processes taking place in the reactor with the stirrer can be written down as a system of equations

$$
\begin{gathered}
\varepsilon \frac{\partial c}{\partial t}+v \frac{\partial c}{\partial x}=-(1-\varepsilon) \gamma k_{0} \frac{3 c(t, x)}{r(t)}, 0<x<L(t) \\
c(t, 0)=c_{0} \\
c(0, x)=0 \\
\frac{\mathrm{d} r}{\mathrm{~d} t}=-\frac{b}{L(t)} \int_{0}^{L(t)} \frac{c(t, x)}{c_{0}} \mathrm{~d} x, b=\gamma k_{0} \frac{\rho_{\mathrm{Y}} M_{\mathrm{Me}}}{\rho_{\mathrm{Me}} M_{\mathrm{Y}}} \\
r(0)=r_{0} \\
\frac{L(t)}{L_{0}}=\left[\frac{r(t)}{r_{0}}\right]^{3} \\
L(0)=L_{0} .
\end{gathered}
$$

In this system of Equations (7)-(9) the processes of sorption / mass transfer are described by Equation (7). The law of the decrease of the granule size is presented by Equation (8), where the coefficient $b$ has a different form (Appendix, formula A5) than in Equation (5). The correlation between the height of the column and the size of the granules is given in Equation (9).

\section{Model Analysis}

Let us introduce the dimensionless variables and functions: time $\tau=t k_{0} / r_{0}$, axial vertical coordinate $\xi=x / L_{0}$, the height of the column of the granules $l(\tau)=L(t) / L_{0}$, the share of the impurity in the gas flow $u(\tau, \xi)=c(t, x) / c_{0}$, the radius of the granules $s(\tau)=r(t) / r_{0}$ and gas purification parameters 
$A=k_{0} L_{0} / v r_{0}$ and $B=\rho_{\mathrm{Y}} M_{\mathrm{Me}} / \rho_{\mathrm{Me}} M_{\mathrm{Y}}$. Then Equations (7)-(9) acquire the following form:

$$
\begin{gathered}
\varepsilon \frac{\partial u}{\partial \tau}+\frac{1}{A} \frac{\partial u}{\partial \xi}=-3(1-\varepsilon) \gamma \frac{u(\tau, \xi)}{s(\tau)}, 0<\xi<l(\tau) \\
u(\tau, 0)=1 \\
u(0, \xi)=0 \\
\frac{\mathrm{d} s}{\mathrm{~d} \tau}=-\frac{B \gamma}{l(\tau)} \int_{0}^{l(\tau)} u(\tau, \xi) \mathrm{d} \xi \\
s(0)=1 \\
l(\tau)=s(\tau)^{3} .
\end{gathered}
$$

The problem (10)-(12) can be solved with the help of the method of two-time scales. In fact, owing to the smallness of $c_{0}$ and the high rate of capturing the gas impurity by the metallic regions of the surface of the granules the smallest time scale is inherent in the function $u(\tau, \xi)$. The distribution of the impurity along the axis of the column adjusts to the changes in the system gas/metal much faster than the other time dependent values.

The second time scale is connected with the characteristic time of the surface rubbing of an individual granule. It is so much bigger than the first one that in the solution of Equation (10) it is possible not to take into consideration the dependence of $s$ on $\tau$, and then we get for the function $u(\tau, \xi)$ the following approximate result:

$$
u(\tau, \xi)=\exp \left[-\frac{3(1-\varepsilon) A \gamma}{s(\tau)}\right]
$$

Further, to solve Equation (11) it is necessary to evaluate the integral

$$
\begin{aligned}
\int_{0}^{l(\tau)} u(\tau, \xi) \mathrm{d} \xi & =\int_{0}^{l(\tau)} \exp \left[-\frac{3(1-\varepsilon) A \gamma}{s(\tau)} \xi\right] \mathrm{d} \xi \\
& =\frac{s(\tau)}{3(1-\varepsilon) A \gamma}\left\{1-\exp \left[-\frac{3(1-\varepsilon) A \gamma}{s(\tau)} l(\tau)\right]\right\} .
\end{aligned}
$$

Substituting into (14) the value of $l(\tau)$ according to Equation (12) we come to

$$
\begin{gathered}
\frac{\mathrm{d} s}{\mathrm{~d} \tau}=-\frac{B}{3(1-\varepsilon) A s(\tau)^{2}}\left\{1-\varepsilon\left[-3(1-\varepsilon) A \gamma s(\tau)^{2}\right]\right\} \\
s(0)=1 .
\end{gathered}
$$

The analytical solution of Equation (15) can be given implicitly

$$
\tau=\frac{3(1-\varepsilon) A}{B} \int_{s(\tau)}^{1} \frac{s^{2}}{1-\exp \left[-3(1-\varepsilon) A \gamma s^{2}\right]} \mathrm{d} s .
$$

Plots in Figure 2 show numerically calculated changes of impurity concentration 


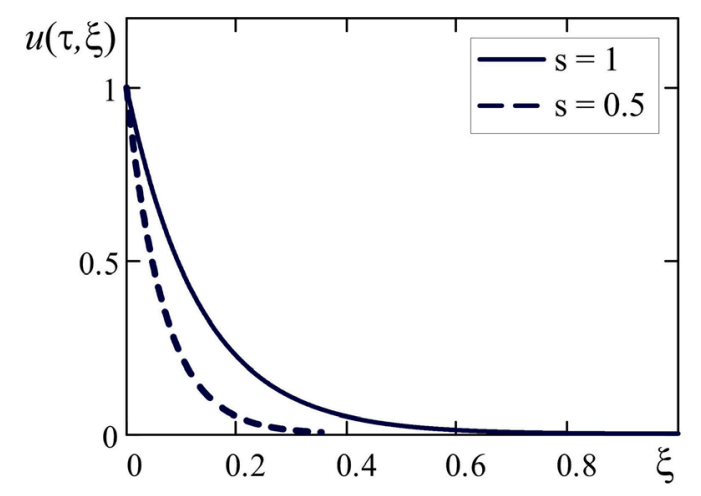

Figure 2. The distribution of impurity concentration in the gas flow along $\xi$ axis in the column of granules Me. $u(\tau, \xi)$ is fraction of impurity $\mathrm{Y}$ in the gas phase, $\tau$-time, $s$-granule radius.

in the gas flowing through the column of stirred granules.

The more intensive the gas purification process, the steeper the curve $u$ falls to 0 . It follows from the formula (13) that it is possible to enhance the intensity of the gas treatment by increasing the values of $\gamma$ and/or $A=L_{0} k_{0} / v r_{0}$. This is exactly what Figure 2 demonstrates: from the two curves $u$ the dash-line one, which corresponds to the granules of the smaller size, is closer to the vertical axis. This is evident as with the decrease of the granules radius the specific surface area of the reactant grows and together with it the reaction kinetics in the system $\mathrm{Me} / \mathrm{Y}$ grows as well.

While Figure 2 shows how the concentration $u$ of the impurity in the gas flow decreases with the approximation to the coordinate $\xi=1$, i.e. to the exit from the column of granules Me, Figure 3 shows how the concentration $u$ of the impurity grows with time $\tau$ in the cross section $\xi=1$.

It is seen also that $u(\tau, 1)$ continues to stay close to zero for a fairly long time after which it starts to increase rapidly. The moment of time $\tau_{c}$ when $u(\tau, 1)$ attains the limit $u_{c}$ of the maximum acceptable impurity concentration $\mathrm{Y}$ in the gas product is critical for the production processes using the sorber as the source of pure gas.

Two $u(\tau, 1)$ curves for different values of $\gamma$ depending on the stirring rate $\omega$ are compared in Figure 4. As may be expected, the degree of gas purification is the higher the larger the share of the free surface of the granules; however at this the consumption of the mechanical energy for the activation of the reactant increases. Finally, Figure 5 provides information on the process kinetics from the dimensional viewpoint.

First, it can be seen that the height of the column Me, described by the dash-line curve, decreases with time linearly almost to zero, where only by the very end of the process the increment of decrease somewhat slows down. Second, the solid-line curve shows that by the moment when the process is over the radius of the granules decreases approximately by five times with respect to the initial value. The radius of the openings in the dividing mesh 5 (Figure 1) is adjusted to 
the value of $\sim 0.2 r_{0}$. So, at the finishing stage of gas purification the unreacted remains of the reactant fall into the waste collector 7 .

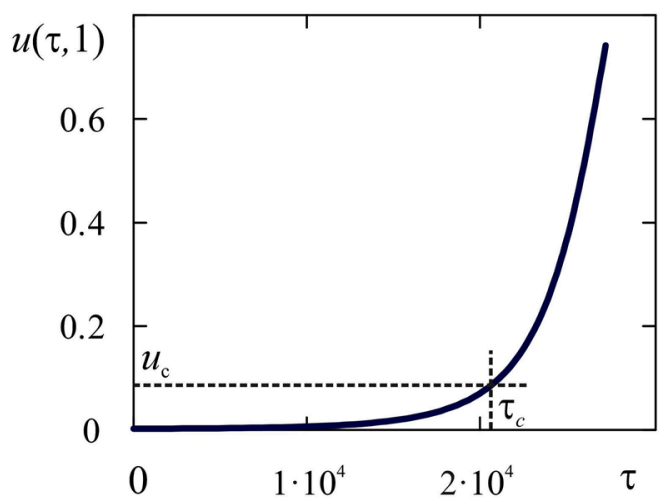

Figure 3. Time dependence of the purity of the end-gas-product. $u(\tau, 1)$-fraction of impurity in outlet gas, $u_{c}$ - the maximally acceptable concentration of impurity in gas product, $\tau$-time, $\tau_{c}$-critical moment in gas purification process.

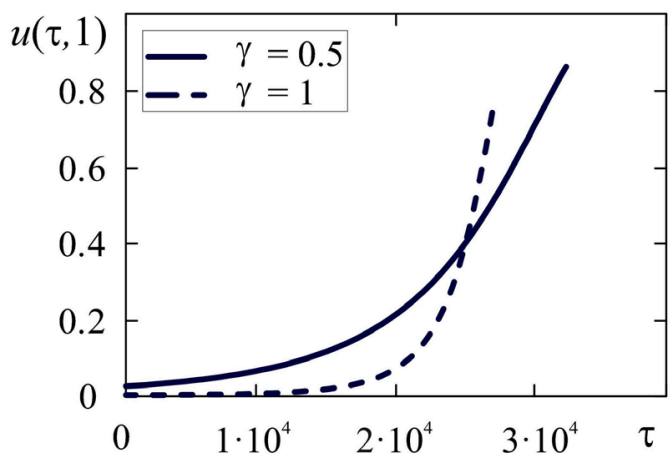

Figure 4. The influence of the stirring intensity on the shape of the curve $u(\tau, 1) . \gamma$-the share of free surface of the granules.

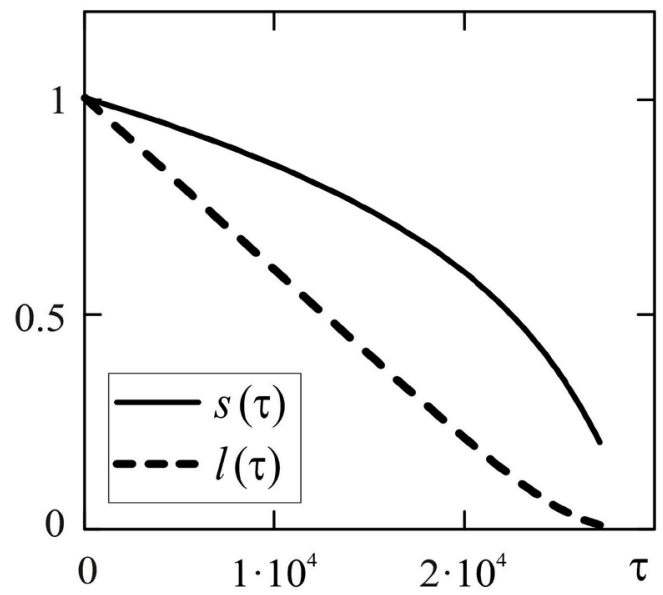

Figure 5. Characteristic behavior of the granules size $s$ and the height $l$ of the column Me with time. 
This organization of the sorption process is rather useful. From the one hand, the losses of the purification material are here negligibly small because the mass of the unspent remains of Me does not exceed $\sim 0.8 \%$ of the initial mass of the reactant. On the other hand, relatively large openings in the dividing mesh make it easy to remove micro particles $\mathrm{MeY}$ from the reaction zone and thus enhance purification kinetics.

\section{Discussion}

Let us consider the consequences of the model, which can be useful for the further development of the technology of tribochemical gas purification by reactants. Mechanical rubbing of the reactants in gas media as a gas purification method is making only the first steps and it is the right time to put the technical recommendations, which follow from the analysis of the causal relations between the production parameters, into engineering solutions. Particularly important is to consider the efficiency of the purification process, under which we understand the specific capturing capacity $q / m_{\mathrm{Me}}$, where $q$ is the amount of the captured gas and $m_{\mathrm{Me}}$ is the mass of the chemisorbent.

Our analysis is built on the comparison of metallic chemisorbents of two different classes, traditional getters adsorbents on the basis of transition metals and getters reactants on the basis of alkali-earth metals. Sorption capacity $q$ of adsorbents is limited by the surface area, which is available for gases. After the surface is saturated with gas the process stops (the passivation state). In this passivated state the getter appears to be by the moment when its installation in the working place is completed. Therefore, it has to be activated in vacuum or in inert medium by heating. At this procedure the atoms of the sorbed gas dissolve in the volume of the getter material purifying in this way its surface and restoring its ability to react with the next portion of gas [17].

One of the most spread methods of production of super pure gases is purification of the gas stream by gas purifiers containing getters adsorbents in powder form or as high porous solid bodies [18] [19] [20] [21] [22]. These bodies are also manufactured by sintering of the powder particles, so it is possible to introduce here a common dimensional characteristic of an adsorbent in a form of an average radius $r$ of the initial getter particle. During the long years of developing of getter technologies approximately the following size range has established in the production of metallic chemisorbents: $10 \mu \mathrm{m} \leq r \leq 200 \mu \mathrm{m}$.

The specific sorption capacity of getters adsorbents in the mentioned size range is very small, approximately by three orders of magnitude lower than the limit, which could be expected taking into account the stoichiometry of the products forming the passivation film. The given fact has provided the impetus for substitution of getters adsorbents for powder reactants in gas purifiers [23]. However, the success of this substitution appeared to be partial: although the sorption capacity of the purification material increased at this by more than an order of magnitude it still remained sufficiently lower than its theoretical limit. 
Further, while in the air the adsorbents just passivate the powder reactants inflame and burn. For this reason the need has been recognized to develop special equipment for transporting reactive powders from vacuum conditions into the gas purifier [23]. The other side of the problem is related with unloading the purifier and utilization of the waste material containing large amount of unreacted metal.

\subsection{The Slowing Layer $\Delta h$}

The answer to the question why such active material as reactant Me does not have enough time to react with the impurity to completion during the time that the gas passes through the gas purifier, is given by the kinetics of the given process. Alkali-earth metals and their alloys in the interaction with gases follow the parabolic law [9] [17] [24]. As the reaction front moves into the volume of the material the rate of capturing gases decreases. Sooner or later it decreases to such an extent that if we are talking about the production process its continuation does not make sense and it should be stopped. The layer MeY of the certain thickness $\Delta h$, which we call here a slowing layer, answers this moment. We can widen the notion of the slowing layer understanding under it such a layer $\Delta h$, which during some operational period of time with the exposure of the granules in the air will increase in the thickness only by a negligibly small value compared with the initial value $\Delta h$. It is possible to estimate the order of magnitude of the value $\Delta h$ for reactants with the help of granulometric and sorption data on Ba-alloys [3] [8] [25] and on ternary alloys Ca-Li-Mg [9]. Using this data we come to the ratio $2 \mu \mathrm{m} \leq \Delta h \leq 5 \mu \mathrm{m}$, which gives us the possibility to define the losses of $\mathrm{Me}$ in the purification of gas by gas purifiers for different values of $r$ of the reactant with the monolithic structure. For the estimation of the share $\eta$ of the unreacted Me a simple formula $\eta=[(r-\Delta h) / r]^{3}$ can be used because the densities of $\mathrm{Me}$ and $\mathrm{MeY}$ do not differ sufficiently from each other.

The accordingly calculated results for $\eta$ at $\Delta h=5 \mu \mathrm{m}$ are collected in Table 1 and depicted in Figure 6.

Table 1. The share $\eta$ of the reactant Me in the production waste at different values of $r$.

\begin{tabular}{ccccccccc}
\hline$r, \mu \mathrm{m}$ & 5 & 10 & 25 & 75 & 200 & 500 & 1000 & 1500 \\
\hline$\eta, \%$ & 0 & 12.5 & 51.2 & 81.3 & 92.7 & 97.0 & 98.5 & 99.0 \\
\hline
\end{tabular}

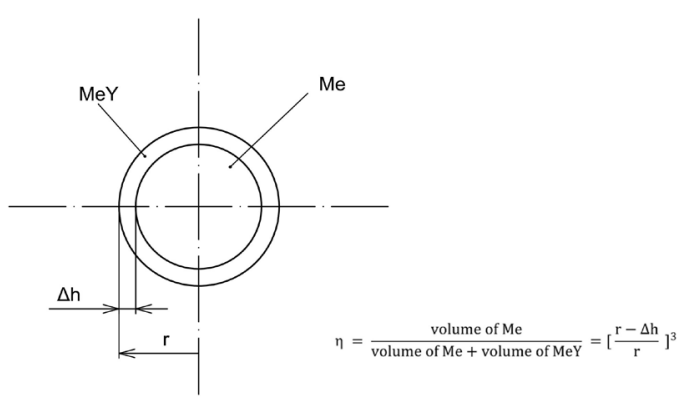

Figure 6. The slowing surface layer. $r$-initial granule radius, $\Delta h$-thickness of slowing MeY layer, $(r-\Delta h)$-radius of residual Me, $\eta$-share of unreacted metal. 
Let us mention an ambiguous role of the cover layer $\Delta h$ in sorption applications. This layer, after it has been formed practically stops the gas purification process in usual sorption columns like gas purifiers but the same layer fulfils a number of useful functions in other cases. So, the value $\Delta h$ of the layer $\mathrm{MeY}$ gives us the knowledge about the amount of the sorbed gas. Further, the presence of the layer of $\mathrm{MeY}$ of the thickness of $\Delta h$ on the monolithic particles of the reactant is the guarantee of the safety of these particles at the contact with the air. It can be said that the layer $\Delta h$ is a sort of an analogue to the passivating film of the getters adsorbents. It is the layer $\Delta h$ that allows loading relatively large particles of the reactant into the tribochemical reactor in air conditions without lowering the sorption potential of the purification material provided the loading procedure is not very lengthy.

It follows from Table 1 that the powder area with particles of the reactant of the size $r \geq 20 \mu \mathrm{m}$ is not suitable for being used in gas purifiers because the share $\eta$ of the unconsumed Me in this case is large. At the same time the data in this table provides the data to such easy and convenient way of filling tribochemicals reactors with purification material as charging them in the air with monolithic particles, the size of which satisfy the condition $r \geq 1.5 \mathrm{~mm}$.

The latter fact gives the reactants sufficient advantages over the adsorbents in the economic respect. Traditional getters on the basis of high melting transition metals before being introduced into the gas purifier undergo a long production path of the multistage treatment of the initial metal or alloy to become high porous and permeable for gases structure. In this context filling the sorber with monolithic particles or cast granules of the reactant Me in the air during one or few minutes looks maximally simple. Such a reactant is in the essence raw material, which without passing through the number of the preliminary production stages directly turns into an active gas sorbent on the instant when the stirrer starts rotating in the sorber.

\subsection{Ideal Sorption Technology}

The physical and chemical properties of the reactants are very different from the properties of the other chemisorbents, so adjusting reactants Me to the current production processes would require with sufficient expenses. At the same time big advantages of the reactants over adsorbents both in sorption and in cost respect make it quite natural to set an objective of developing a gas purification technology, which will be initially oriented towards getters reactants.

The best solution appears to be in an integration of getter reactants with the tribochemical reactor, where the first ones serve as consumable materials and the second one performs permanent activation of the getter. Due to such integration the highest level of simplification of the production procedures, their extreme profitability and efficiency can be reached. Let us demonstrate this.

Low mechanical strength of alkali-earth metals and the cover layers of MeY in combination with the brittleness of the latter explains easy rubbing of the gra- 
nules. Due to this property it is possible to remove, layer after layer, the outgrowths of MeY, which is forming on the surface of the granules, with minimal power consumption and in this way to provide unimpeded access of gases to Me. As the result the total sorption surface of the reactants appears to be by orders of magnitude higher than the surface area of any getters adsorbents of the same mass.

The principle difference of tribochemical gas purification from the current methods and its undoubted advantage is the controllability of the sorption process: the intensity of reactions between the chemisorbent and the gas in the sorber is regulated by rotating speed $\omega$ of the stirrer, which determines the share of the free from MeY surface of the granules. As it is shown by the model, at constant value of $\gamma$ the linear or almost linear time law of lowering the height $l(\tau)$ is established, which is demonstrated in Figure 7. According to this law, which in the dimensional form is written as $L(t)=L_{0}-z t$, where $z$ is the proportional coefficient, it becomes easier to select the more economic production regime.

This selection in the cases, when the sorber serves directly the end user of gas, is made by comparing two values, the amount of the gas product with the purity not lower than the set level $u_{c}$ and the power consumption, which depend on $\omega$ and $t$, where $0 \leq t \leq r_{0} \tau_{\mathrm{c}} / k_{0}$ (Figure 3). Using the linear law, we find for any moment of time $t$ such values as the amount of the consumed reactant, equal to $\rho_{\mathrm{Me}}(1-\varepsilon) \pi R^{2} z t$, its current sorption capacity $q / m_{\mathrm{Me}}=\left(z M_{\mathrm{Y}} / L_{0} M_{\mathrm{Me}}\right) t$, the amount of treated gas $v \pi R^{2} t$, and the current rate of capturing gases $z M_{\mathrm{Y}} / M_{\mathrm{Me}} L_{0}$, which is a constant if the speed $\omega$ is constant. The mentioned data is enough for the price comparison of the end product and the production costs, which makes it possible to take an optimal decision.

However, we can go still further. Figure 8 depicts the evolution of the granular column Me in a form of a sequence of four states.

The initial position (a) shows the column at $t=0$, when its height is equal to $L_{0}$. After some rubbing of the granules during the time $t$ the height of the column Me decreases to the level of $L(t)=L_{0}-z t$ (position (b)). Further, position (c) answers the critical moment of time $t=t_{\mathrm{c}}$, which appears in any applications

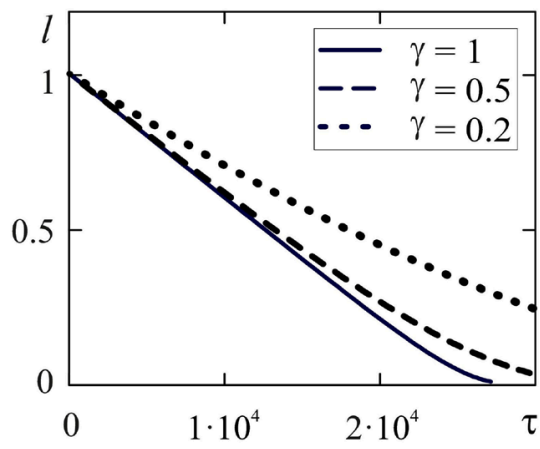

Figure 7. Dependence of function $l(\tau)$ on parameter $\gamma$. 


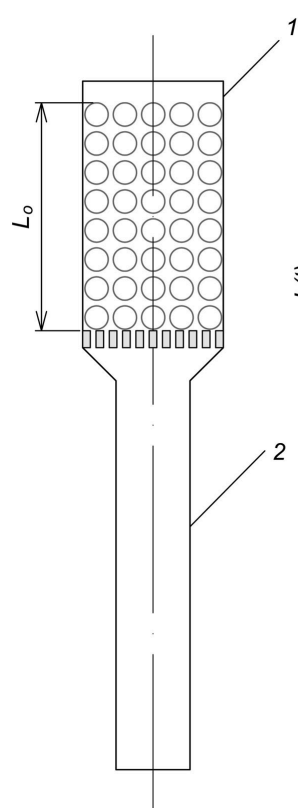

(a)

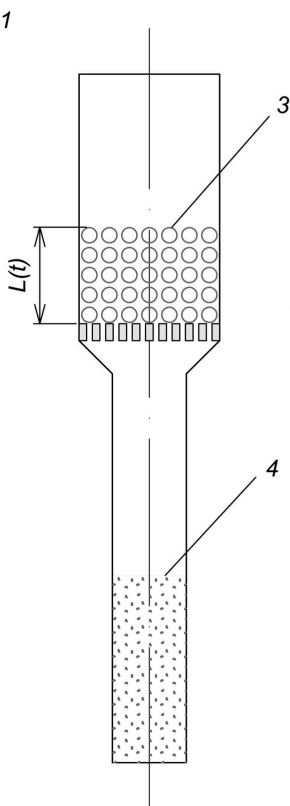

(b)

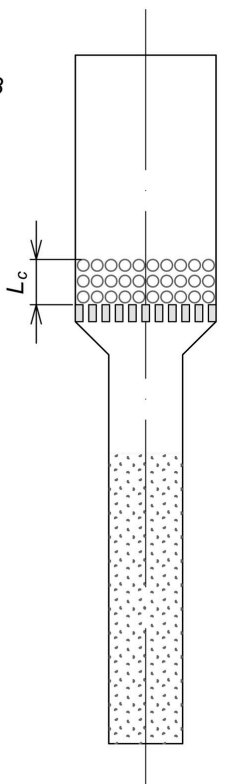

(c)

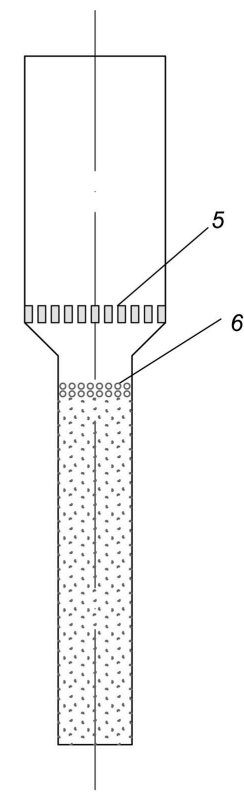

(d)

Figure 8. The changes in column $\mathrm{Me}$ as far as the granules are milled. (a) Initial state, 1 -reactor, 2-waste collector; (b) Intermediate state at the moment of time $t>0$, when the height of the column Me is $L(t)$, 3-granules of the reactant, 4-waste MeY; (c) The state at the moment $t_{\mathrm{c}}$, when the purity of the outlet gas reaches the critical level $u_{\mathrm{c}}$; (d) End of the process, which takes place at the decrease of the radius of the granules smaller than $0.2 r_{0}, 5$-dividing mesh, 6-metallic remains of $\mathrm{Me}$ in the waste.

of the sorber, where the latter is a part of the process system with limitations of the type $u \leq u_{\mathrm{c}}$ in respect to the impurity for the employed gas (Figure 3). Finally, position $(d)$ presents the final stage of rubbing of the granules, when their size reduces so much that they all fall into the waste collector through the openings of the dividing mesh 5 with the diameter of $\sim 0.2 r_{0}$ (Figure 8).

According to Figure 8 in the applications, where the sorber purifies gas, which flows from one tank to another, the tribochemical process of the gas flow treatment can last till Me is completely consumed (position $(d)$ ). The losses of the reactant here are very small, about $0.8 \%$, and examples of this kind of technology can be recycling processes for gases like helium, neon, xenon, etc. If the sorber is built in into the production system, where the purity of the employed gas cannot be lower than $u_{c}$, then the share of the losses of the reactant (as is seen from position (c)) is equal to $\eta_{\mathrm{c}}=L_{\mathrm{c}} / L_{0}$. In the frames of the model under discussion it is possible to reduce these losses by shifting the point $t_{\mathrm{c}}$ towards higher values but this is achieved by the price of increasing the speed of stirring the granules $\omega$.

In the given situation, it is possible to reduce the value of $\eta_{\mathrm{c}}$ without increasing the energy costs only by radical restructuring of production conditions. As it is seen from Figure 8 this possibility exists: if in position (b), which corresponds to the moment of time $t$, we fill the sorber with granules Me to the initial level $L_{0}$ without interrupting the working process, then the critical moment $t_{\mathrm{c}}$ 
will take place later by the mentioned value $t$, i.e. at $t_{\mathrm{c}}+t$. Then, after this time $\left(t_{\mathrm{c}}+t\right)$ passes, it is possible to repeat this procedure again and again turning in this way the sorber from the apparatus of periodical operation into a continuously operating apparatus. It is preferable to carry out this kind of additional feed of the column Me, starting from small values of $t$, for example, as soon as the initial level of the column $L_{0}$ goes down by the value a little more than $2 r_{0}$.

The time dependence of the share of losses of Me in this renewed variant of the process can be estimated with the help of the expression $\eta(t)=L_{\mathrm{c}} /\left(L_{0}+z t\right)$, from where it is seen that $\eta(t)$ is smaller than $\eta_{\mathrm{c}}$ and this difference with time will only grow. Finally, when the given apparatus settles into a stationary operating regime the value $\eta$ approximates the same $0.8 \%$ as in position (d). Figure 9 shows schematically what a reactive sorber of continuous operation may look like.

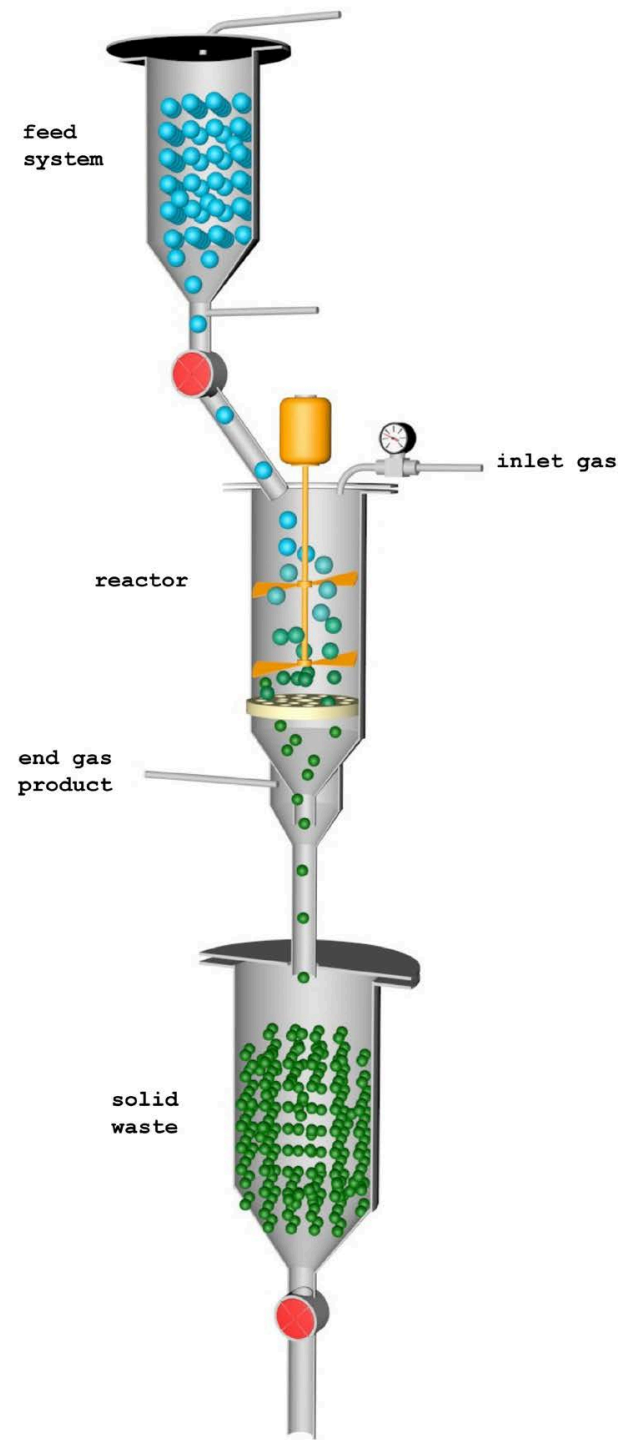

Figure 9. A schematic view of a reactive sorber of continuous operation. 
In the given publication we do not provide a detailed description of the design of the sorber of continuous operation, however it is seen from the above said that the new solution looks ideal: the content of the unreacted share of the reactant is equal to the estimated $0.8 \%$; unloading of the waste of this composition from the sorber is safe as well as filling the sorber in the air with granules of $\mathrm{Me}$ with the size $r \geq 1.5 \mathrm{~mm}$, all that under the condition that the technological requirement to the gas product in the form of $u \leq u_{\mathrm{c}}$ is fulfilled.

Tribochemical gas purification by alkali earth reactants has no limitations. It is applicable in manufacturing of super pure gases, in recycling of rare gases, in purification of natural gases from moisture, mercury vapor, nitrogen, sulfur-and oxygen containing impurities, etc.

\section{Conclusions}

1) The mathematical model of the sorption process in the flow tribochemical reactor with a stirrer has been built for the systems gas/solid, where the solid is monolithic granules of reactant Me consisting of alkali-earth metals or their alloys.

2) According to the solutions of the model the main characteristics of the processes of mass transfer in the sorber at $\omega=$ const linearly depend on time and this fact sufficiently simplifies the control of the production process.

3) The problem of maximizing the sorption efficiency of the reactants Me in the processes of production of pure gases is solved differently in different applications. In tribochemical reactors while the purifying gas flows from one gas tank into another, the share $\eta$ of losses of Me is set by the diameter of the openings in the dividing mesh and in the case when these openings have the size of $0.2 r_{0}$ the sorption result is close to the theoretical limit being inferior to it by less than $1 \%$.

4) In the production systems using the gas of the purity $u \leq u_{\mathrm{c}}$, the purification process can be optimized by adjusting the speed $\omega$ to a value, at which the total expenses for the material Me and for the mechanical work are minimal.

5) The slowing layer MeY of the thickness $\Delta h$ fatally decreases the sorption kinetics and together with it the production efficiency of getter reactants in flow sorption columns of gas purifier type but this layer is useful in tribochemical reactors as it fulfills a protective function during the filling the reactor with reactant $\mathrm{Me}$ in the air.

6) An ideal solution to the problem of sorption efficiency is the substitution of the tribochemical reactor of periodic operation for the reactor of continuous operation. In the latter the total share $\eta$ of losses of reactant Me decreases with time to the level lower than $1 \%$ even at small speeds of $\omega$.

\section{Conflicts of Interest}

The authors declare no conflicts of interest regarding the publication of this paper. 


\section{References}

[1] Littmann, M. (1938) Getterstoffe und ihre Anwendung in der Hochvacuumtechnik. CF Wintersche Verlagshadlung, Leipzig.

[2] Pirani, M. and Yarwood, J. (1961) Principles of Vacuum Engineering. Reinhold Publishing Corporation, New York.

[3] Boffito, C. and Schiabel, A. (1994) Process for the Sorption of Residual Gas by Means of Non-Evaporable Barium Getter Alloy. US Patent No. 5312606.

[4] Ferrario, B. (1996) Chemical Pumping in Vacuum Technology. Vacuum, 47, 363-370. https://doi.org/10.1016/0042-207X(95)00252-9

[5] Chuntonov, K.A. and Yatsenko, S.P. (2013) Getter Film for Small Vacuum Chambers. Recent Patents on Materials Science, 6, 29-39. https://doi.org/10.2174/1874464811306010029

[6] Chuntonov, K. (2013) Safe Gas Sorbents with High Sorption Capacity on the Basis of Lithium Alloys. US Patent No. 8529673.

[7] Fransen, J.J.B. and Perdijk, H.J.R. (1960) The Absorption of Gases by Barium Getter Films Applied as a Tool. Vacuum, 10, 199-203.

https://doi.org/10.1016/0042-207X(60)90136-6

[8] Chuntonov, K. and Setina, J. (2016) Reactive Getters for MEMS Applications. Vacuum, 123, 42-48. https://doi.org/10.1016/j.vacuum.2015.10.012

[9] Chuntonov, K., Ivanov, A.O., Verbitsky, B. and Setina, J. (2018) Getters for Vacuum Insulated Glazing. Vacuum, 155, 300-306.

https://doi.org/10.1016/j.vacuum.2018.06.012

[10] Chuntonov, K., Atlas, A., Setina, J. and Douglass, G. (2016) Getters: From Classification to Materials Design. Journal of Materials Science and Chemical Engineering, 4, 23-34. https://doi.org/10.4236/msce.2016.43004

[11] Chuntonov, K., Verbitsky, B., Ivanov, A.O. and Kozhevnikov, V.L. (2018) Mechanochemical Methods in the Production of High Purity Gases. Materials Sciences and Applications, 9, 489-501. https://doi.org/10.4236/msa.2018.95034

[12] Heinike, G. (1984) Tribochemistry. Akademie-Verlag, Berlin.

[13] Butyagin, P.Y. (1986) Mechanochemical Reactions of Solids with Gases. Reactivity of Solids, 1, 345-359. https://doi.org/10.1016/0168-7336(86)80026-2

[14] Kajdas, C. and Hiratsuka K. (2018) Tribocatalysis, Tribochemistry, and Tribocorrosion. Taylor \& Francis, Pan Stanford Publishing, New York.

https://doi.org/10.1201/b20123

[15] Bolm, C. and Hernandez, J.G. (2019) Mechanochemistry of Gaseous Reactants. Angewandte Chemie International Edition, 58, 3285-3299. https://doi.org/10.1002/anie.201810902

[16] Chuntonov, K., Ivanov, A.O., Verbitsky, B. and Kozhevnikov, V.L. (2017) Gas Purification and Quality Control of the End Gas Product. Journal of Materials Science and Chemical Engineering, 5, 44-58.

[17] Ferrario, B. (1998) Getters and Getter Pumps. In: Lafferty, J.M., Ed., Foundations of Vacuum Science and Technology, Wiley, New York, 261-316.

[18] Kohl, A.L. and Nielsen, R.B. (1997) Gas Purification. 5th Edition, Gulf Publishing Company, Houston, TX.

[19] Schön, H. (2015) Handbook of Purified Gases. Springer-Verlag, Berlin. https://doi.org/10.1007/978-3-540-32599-4 
[20] Murphy, W., Edlung, E. and Brown, K. (2008) Purifier. US Patent No. 7438864.

[21] Entegris (2018) White paper. Bowling for Contaminants: The New Science of Gas Purification. 1-7.

https://info.entegris.com/white-paper-bowling-for-contaminants?utm_campaign= Gas\%20Purity\&utm_source=entegris_website\&utm_medium =campaign_page\&ut $\underline{\text { m_content }=\text { whitepaper }}$

[22] Entegris (2019) White Paper. Eliminating Unwanted Oxygen: Preventing Device Failure at the Source. 1-6.

https://info.entegris.com/eliminating-unwanted-oxygen-preventing-device-failure-a t-the-source

[23] Chuntonov, K. and Setina, J. (2017) Activationless Gas Purifiers with High Sorption Capacity. US Patent No. 9586173.

[24] Ricca, F. and della Porta, P. (1960) Carbon Monoxide Sorption by Barium Films. Vacuum, 10, 215-222. https://doi.org/10.1016/0042-207X(60)90140-8

[25] Schiabel, A. and Boffito, C. (1994) Process for the Sorption of Residual Gas by Means by a Non-Evaporated Barium Getter Alloy. US Patent No. 5312607. 


\section{Appendix}

Let us consider an elementary layer $\mathrm{d} x$ of a cylindrical column of granules Me. Let $R$ be the radius of this column and $r$ be the radius of the granules. Then the volume of the elemental layer is $V=\pi R^{2} \mathrm{~d} x$, the number of granules in it is $n=3(1-\varepsilon) R^{2} \mathrm{~d} x / 4 r^{3}$, and the surface area of these granules is equal to $S_{\mathrm{Me}}=3 \pi(1-\varepsilon) R^{2} \mathrm{~d} x / r$. Let us calculate the consumption $\mathrm{d} m_{\mathrm{Me}}$ of the reactant during the time $\mathrm{d} t$ for capturing the impurity $\mathrm{Y}$ in the reaction $\operatorname{Me}(\mathrm{s})+\mathrm{Y}(\mathrm{g})=\operatorname{MeY}(\mathrm{s})$, where (s) means the solid state and (g) means the gaseous state. This consumption can be calculated by multiplying the atomic mass $M_{\mathrm{Me}}$ of the reactant by the number $\mathrm{d} N_{\mathrm{Y}}$ of the molecules $\mathrm{Y}$ the captured by the surface $S_{\mathrm{Me}}$.

As the second factor is

$$
\mathrm{d} N_{\mathrm{Y}}=\gamma k_{0} S_{\mathrm{Me}}(t) c(t, x) \frac{P}{k_{\mathrm{B}} T} \mathrm{~d} t
$$

then, substituting the value of $S_{\mathrm{Me}}$ and multiplying the result by $M_{\mathrm{Me}}$, we get

$$
\mathrm{d} m_{\mathrm{Me}}=\frac{3 \pi(1-\varepsilon) \gamma k_{0}}{r(t)} c(t, x) \frac{P R^{2}}{k_{\mathrm{B}} T} \frac{M_{\mathrm{Me}}}{N_{\mathrm{A}}} \mathrm{d} x \mathrm{~d} t,
$$

where $N_{\mathrm{A}}$ is the Avogadro number. However, the same loss of the mass $\mathrm{Me}$ can be expressed in another way, via the reduction of the radius of the metallic part of the granule by the value $\mathrm{d} r$ during the mentioned time $\mathrm{d} t$

$$
\mathrm{d} m_{\mathrm{Me}}=\frac{4 \pi n \rho_{\mathrm{Me}}}{3}\left[r^{3}-(r-\mathrm{d} r)^{3}\right]=4 \pi n \rho_{\mathrm{Me}} r^{2} \mathrm{~d} r .
$$

Substituting now the value $n$ into (A3) and setting the obtained expression to (A2) we get finally

$$
\frac{\mathrm{d} r}{\mathrm{~d} t}=-b \frac{c(t, x)}{c_{0}}, \quad b=\gamma k_{0} c_{0} \frac{M_{\mathrm{Me}}}{N_{\mathrm{A}} \rho_{\mathrm{Me}}} \frac{P}{k_{\mathrm{B}} T} .
$$

The coefficient $b$ can also be converted to a simpler form

$$
b=\gamma k_{0} \frac{\rho_{\mathrm{Y}} M_{\mathrm{Me}}}{\rho_{\mathrm{Me}} M_{\mathrm{Y}}},
$$

where $\rho_{\mathrm{Y}}$ and $M_{\mathrm{Y}}$ are the mass density of impurity $\mathrm{Y}$ in gas flow $c_{0}$ and the molar mass of the impurity $\mathrm{Y}$ accordingly. 